\title{
Toward Carbon Nanotube-Based AFM Cantilevers
}

\author{
Jianhua Tong, Member, IEEE, and Yu Sun, Senior Member, IEEE
}

\begin{abstract}
The mechanical properties of carbon nanotubes have been widely employed to enhance the performance of atomic force microscopy (AFM) cantilever tips. Utilizing the electromechanical properties of carbon nanotubes, this paper investigates the potential of using carbon nanotubes as active strain sensing elements on AFM cantilevers. A batch microfabrication process was developed to construct silicon microcantilevers. Multiwalled carbon nanotubes were dielectrophoretically assembled between electrodes. Based on the characterization results of 12 devices, the CNT-based cantilevers demonstrated a linear relationship between resistance changes and externally applied strain. The gauge factor ranged from $\mathbf{7 8 . 8 4}$ to $\mathbf{1 3 4 . 4 0}$ for four different device configurations.
\end{abstract}

Index Terms-Atomic force microscopy, cantilevers, carbon nanotubes, MEMS/NEMS (micro/nanoelectromechanical systems).

\section{INTRODUCTION}

A TOMIC force microscopy (AFM) has been widely applied to investigating surface topographies at the nano scale and imaging/manipulating living biological molecules and cells in their native environments [1], [2]. Besides a piezoelectric motion stage, AFM employs a microelectromechanical systems (MEMS) based cantilever with a sharp tip at its end that is used to scan a specimen surface. The deflection of the cantilever is typically sensed by a laser-photodiode optical system.

As the imaging resolution of an AFM depends on the sharpness of the cantilever tip, efforts have been spent on developing micronanofabrication processes to improve tip sharpness. However, existing micronanofabricated cantilever tips are still limited to tens of nanometers. Although the laser detection system provides a high sensitivity for detecting cantilever deflections, the relatively bulky size makes system integration difficult and restricts its use from applications in which stringent space constraints are a concern [e.g., inside the chamber of a scanning/transmission electron microscope (SEM/TEM)]. Furthermore, when used in aqueous environments where biological materials survive, the reflection and refraction of the transmitted light must be cautiously corrected to minimize the inaccuracy of molecular or cellular force measurements. In summary, it is desired to increase the cantilever tip sharpness down to a few nanometers and replace the external laser detection system with an integrated, electronic "self-sensing"

Manuscript received July 18, 2006; revised May 28, 2007. This work was supported in part by the Materials and Manufacturing Ontario and in part by the Ontario Ministry of Research and Innovation through an Early Researcher Award. The review of this paper was arranged by Associate Editor M. Freeman.

The authors are with the Advanced Micro and Nanosystems Laboratory, Department of Mechanical and Industrial Engineering, University of Toronto, Toronto, ON M5S 3G8, Canada (e-mail: sun@mie.utoronto.ca).

Color versions of one or more of the figures in this paper are available online at http://ieeexplore.ieee.org.

Digital Object Identifier 10.1109/TNANO.2007.901910



Fig. 1. A 3-D solid model of CNT-based AFM cantilever. (a) CNT-enhanced sharp tip. (b) CNT-based strain sensor for deflection detection.

mechanism. On these fronts, carbon nanotubes (CNTs) are capable of providing solutions to both issues.

CNTs exhibit remarkable mechanical properties irrespective of their chirality, including high aspect ratios, great tensile strength, and high resilience [3]. Mechanical properties differ significantly between single-walled carbon nanotubes (SWNTs) and multiwalled carbon nanotubes (MWNTs). The radial Young's modulus of MWNTs was found to strongly decrease with increasing radius [4]. External radii of about $2 \mathrm{~nm}$ show a Young's modulus of $400 \pm 200 \mathrm{GPa}$, dropping off to a value an order of magnitude lower at $30 \pm 10 \mathrm{GPa}$ for external radii $4 \mathrm{~nm}$ or greater. This demonstrates the importance for CNT-based mechanical sensors to use a homogeneous set of CNTs, both of type (SWNT versus MWNT) and of radius, as mechanical properties change dramatically as these variables are altered.

Through either direct in situ growth [5], [6] or postgrowth assembly [7], [8], it has been demonstrated that an individual CNT or a small bundle of CNTs can be attached to a sharp AFM cantilever tip [Fig. 1(a)]. Due to their nanometer size and remarkable mechanical properties of CNTs, the CNT scanning tips significantly increase the probing depth and imaging resolution (e.g., capable of scanning vertical sidewalls up to $87^{\circ}$ [9]).

Due to the potential integration of CNTs in nanoelectromechanical systems (NEMS) based sensors, the effect of mechanical deformation on the electrical properties of CNTs has been of keen interest. Several experimental studies have been conducted, in which an AFM tip was used to locally bend a suspended SWNT [10], [11] or tensile stretching individual SWNT [12] and conductance changes monitored. Depending on the specific nanotube type tested (quasimetallic, semiconducive, or metallic) and how load was applied, conductance changed by $35 \%$ [11] or by orders of magnitude [10], [12]. These electromechanical characterization results serve as the fundamental rationale of developing highly sensitive strain sensors using CNTs as active sensing elements. 
Employing the piezoresistive effect of CNTs, a MEMS pressure sensor that integrated bundles of MWNTs on a PMMA substrate was reported to have a piezoresistive gauge factor of 235 [13]. More recently, a SWNT-based pressure sensor was constructed by electron-beam lithography and a bulk micromachining process [14]. The pressure sensor consisted of an individual metallic SWNT on top of a $100 \mathrm{~nm}$ thick circular alumina membrane with a radius of $50-100 \mu \mathrm{m}$. The piezoresistive gauge factor was characterized to be approximately 210 . The low-throughput e-beam lithography process followed by AFM imaging for alignment and coordinate determination eliminates the potential of batch fabrication.

The piezoresistive properties of CNTs promise a potential candidate for strain sensing in AFM imaging/manipulation to replace the laser detection system [Fig. 1(b)]. In this paper, we present the design and microfabrication of cantilevers that employ MWNTs as active sensing elements, differently from conventional piezoresistive designs using doped silicon or polysilicon. The MWNTs were integrated through dielectrophoresis (DEP) trapping. A batch microfabrication process was developed to construct these CNT-based cantilevers, which is describedg in Section III. Characterization results and discussion are presented in Section IV.

\section{ELECTRODE CONTACT INTEGRATION OF CNTS}

The central idea of nanotube integration is to create electrical contacts between a nanotube and electrodes, representing a critical step for creating nanotube-based devices. A detailed discussion on the topic of CNT integration can be found in [15]. A few of the integration techniques are briefly described as follows. The first method is to disperse CNTs on a substrate and randomly locate a nanotube (or nanotube ropes) using AFM. A subsequent e-beam lithography step creates electrodes to fix the located nanotubes or ropes [14]. The second method employs either e-beam lithography [10], [16], [17] or micro contact printing [18], [19] to pattern catalysts, followed by CVD in situ growth. Another e-beam lithography step is used to place electrical contact pads over the catalyst islands. The third integration technique combines SEM-based nanorobotic manipulation and EBID (electron beam induced deposition) [20].

Among the many CNT integration techniques, DEP manipulation was demonstrated to be an effective approach for the assembly of CNTs onto electrodes [21], [22]. Using a composite dc/ac field, the DEP approach employs the ac field to attract nanotubes to their desired location while using the dc field to trap the nanotubes by electrostatic attraction. Electrode size and geometry, ac frequency, and dc/ac ratio are major factors in determining the alignment and the number of trapped nanotubes.

In our experiments, purified MWNTs (Nanoledge Inc., France) with an average length of $5 \mu \mathrm{m}$ and an average diameter of $17 \mathrm{~nm}$ were used. The MWNTs dispersed and suspended in methanol with Triton X-100 were sonicated (Fisher FS220) at $320 \mathrm{~W}$ for $4.5 \mathrm{~h}$ in order to untangle the MWNT ropes into individual tubes. The concentration of the resulting MWNT solution for DEP integration was $0.5 \mu \mathrm{g} / \mathrm{ml}$. Au/Cr electrodes (Fig. 2) having a thickness of $80 \mathrm{~nm} / 20 \mathrm{~nm}$ and a gap of $2 \mu \mathrm{m}$ were constructed by e-beam evaporation and liftoff. The trapping experiments were conducted over all combinations
TABLE I

DEP TRapping Parameter VAlues. All Combinations Were Conducted

\begin{tabular}{lccc}
\hline \hline \multicolumn{1}{c}{ Factor } & Value 1 & Value 2 & Value 3 \\
& & & \\
\hline Driving frequency (MHz) & 4 & 8 & 12 \\
AC magnitude (V) & 3 & 6 & 9 \\
DC offset (V) & 0.5 & 1 & 1.5 \\
\hline
\end{tabular}

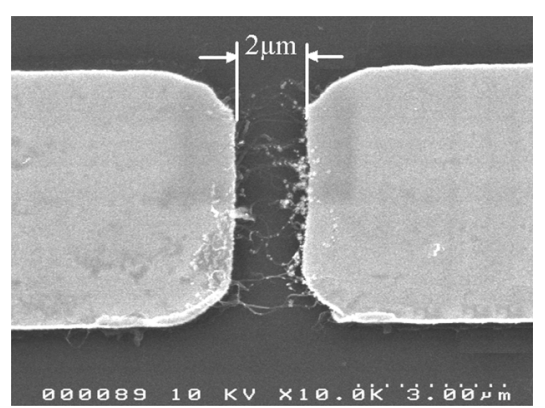

Fig. 2. SEM image of a pair of microelectrodes with MWNTs assembled by DEP trapping. The applied signal consisted of a $4 \mathrm{MHz} / 9 \mathrm{Vp}-\mathrm{p}$ ac component and a $0.5 \mathrm{~V} \mathrm{dc}$ offset.

of parameter values listed in Table I. The trapping parameter set of $4 \mathrm{MHz}$ and $9 \mathrm{Vp}-\mathrm{p}$ ac signal with a $0.5 \mathrm{~V} \mathrm{dc}$ offset was selected for assembling MWNTs onto electrodes because this parameter set was found to produce a regular MWNT network with limited number of tubes (Fig. 2) and result in the highest reproducibility and yield across devices.

After MWNT integration, a final photolithography and liftoff step was performed to deposit $20 \mathrm{~nm}$ thick $\mathrm{Au}$ as top electrodes over the aligned nanotubes. This additional step serves two purposes. First, it dramatically improves the contact resistance between the CNT and the surrounding electrode assembly (decreased from 1-10 M $\Omega$ to $100-600 \mathrm{k} \Omega$ ). Second, it physically "clamps" the nanotubes in place.

\section{MICROFABRICATION}

Microfabrication started with a $400 \mu \mathrm{m}$ thick $\langle 100\rangle$ Si wafer. A $200 \mathrm{~nm} \mathrm{Si} \mathrm{N}_{3} \mathrm{~N}_{4}$ film was formed by LPCVD on both sides. A rectangular window was opened by $\mathrm{Si}_{3} \mathrm{~N}_{4}$ RIE etching, followed by $\mathrm{KOH}$ anisotropic Si etching until $40 \mu \mathrm{m}$ Si remained in the trench [Fig. 3(a)]. Using front-to-back alignment, $80 \mathrm{~nm} / 20 \mathrm{~nm} \mathrm{Au} / \mathrm{Cr}$ electrodes with a gap of $2 \mu \mathrm{m}$ were formed on the front side of the wafer with e-beam evaporation and liftoff [Fig. 3(b)]. In order to investigate the electrode shape effect on cantilever performance, four electrode configurations were constructed, shown in Fig. 4. Round electrodes are used in Fig. 3(c) and (d) to illustrate the fabrication process.

MWNTs were DEP assembled onto microelectrodes under a MEMS testing probe station. After a droplet of MWNT solution $(2 \mu \mathrm{L})$ was placed between a pair of electrodes, the trapping voltage was continuously applied until the MWNT solution evaporated completely. When CNT assembly was completed on all electrode pairs, the wafer was placed onto a hotplate at $100{ }^{\circ} \mathrm{C}$ for $30 \mathrm{~min}$ to remove methanol [Fig. 3(c)].

A second layer of $20 \mathrm{~nm}$ Au electrodes was formed by liftoff, in which a high-precision alignment to the bottom $\mathrm{Au} / \mathrm{Cr}$ electrodes was performed [Fig. 3(d)]. This step was effective in re- 


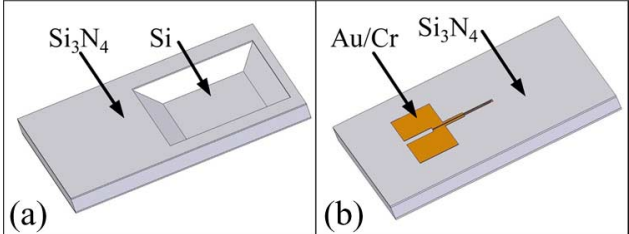

(a)
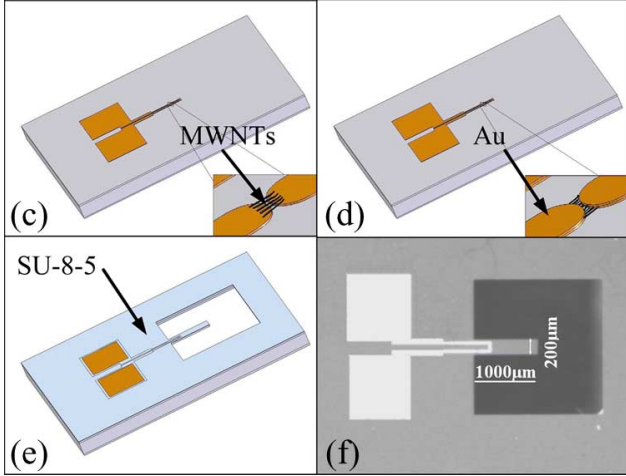

Fig. 3. Microfabrication process flow of MWNT-based cantilevers. (a) KOH Si etching. (b) E-beam evaporation and liftoff. (c) DEP trapping of MWNTs. (d) Liftoff to form top-covering electrodes. (e) SU-8 patterning and dry etching release. (f) Optical microscopy picture of a released cantilever suspended over a trench.

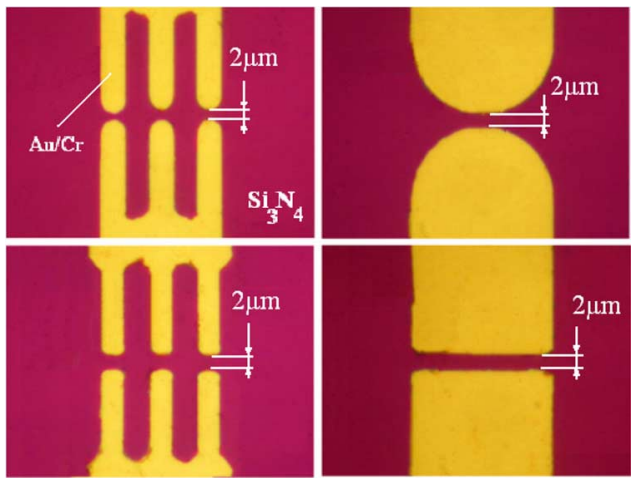

Fig. 4. Optical microscopy pictures of four electrode configurations. (a) Repeated round electrodes. (b) Single round electrodes. (c) Repeated rectangular electrodes. (d) Single rectangular electrodes.

ducing contact resistance and mechanically fixing the MWNTs. The wafer was cleaned with alcohols, rinsed with DI water, and placed onto a hotplate for another $30 \mathrm{~min}$ at $100{ }^{\circ} \mathrm{C}$. A $10 \mu \mathrm{m}$ SU-8-5 negative photoresist was then spun onto the wafer and patterned. This SU-8 layer acted as etch mask for subsequent dry release. Additionally, it served as a packaging layer that separated MWNTs from the external environment for protecting nanotubes during dry etching and minimizing their responsiveness to external environmental changes, such as humidity and gas concentration variations.

Using the patterned SU-8 as an etch mask, a $\mathrm{Si}_{3} \mathrm{~N}_{4}$ RIE etch and a Si DRIE etch released the cantilevers [Fig. 3(e)]. Fig. 3(f) shows an optical microscopy picture of a released CNT-based cantilever suspended over a trench.

\section{Characterization Results and Discussion}

The effective Young's modulus of the cantilever is

$$
E_{\mathrm{eff}}=\frac{E_{\mathrm{Si}} h_{\mathrm{Si}}+E_{\mathrm{SU} 8} h_{\mathrm{SU} 8}}{h_{\mathrm{Si}}+h_{\mathrm{SU} 8}}
$$

where $E_{\text {eff }}$ is the effective Young's modulus; $E_{\mathrm{Si}}=160 \mathrm{GPa}$ and $E_{\mathrm{SU} 8}=4 \mathrm{GPa}$ are the Young's modulus of Si and SU-8; and $h_{\mathrm{si}}=40 \mu \mathrm{m}$ and $h_{\mathrm{SU} 8}=10 \mu \mathrm{m}$ are thickness of Si and SU-8. The force-deflection $(\delta)$ model of the cantilever is

$$
F=\frac{3 \delta E_{\text {eff }} l}{L^{3}}
$$

where $I$ is moment of inertia, and $L=1000 \mu \mathrm{m}$ is the cantilever length.

Testing was conducted with a micromanipulator (Sutter MP 285) under a probe station. As CNTs are temperature sensitive (i.e., a temperature rise results in a lower resistance [23]), room temperature at $25^{\circ} \mathrm{C}$ was maintained throughout the characterization process. A stiff probe was mounted on the three-axis micromanipulator that applied forces to the cantilevers. At every step of $2 \mu \mathrm{m}$, the resistance change was measured with a precision source meter (Keithley 2602). From (1) and (2), cantilever deflections were converted into unitless strain. Fig. 5 shows the testing results (resistance change versus tensile strain) of one representative cantilever for each electrode configuration. In the experiments, three cantilevers for each electrode configuration were characterized (12 cantilevers total).

Experimental results demonstrated that the initial resistance was the highest for repeated round electrodes [Fig. 5(a)] and the lowest for single rectangular electrodes [Fig. 5(d)]. Due to the largest effective area of single rectangular electrodes for CNT trapping, more MWNTs were assembled between two electrodes. As the total resistance is determined by the number of parallel connected CNTs, more assembled CNTs resulted in a lower initial resistance. Despite the initial resistance differences, the slope of the curves for the four electrode configurations was found to be relatively stable, from $1.9 \times 10^{4} \mathrm{k} \Omega$ to $6.5 \times 10^{4} \mathrm{k} \Omega$ (with a mean value of $3.5 \times 10^{4} \mathrm{k} \Omega$ and a standard deviation of $3.56 \times 10^{4} \mathrm{k} \Omega$ ) based on the testing results of 12 cantilevers. Within the testing range, no noticeable hysteresis was observed. For larger stain levels, hysteresis might be significant and must be thoroughly quantified.

As an important measure of CNTs' electromechanical property, the piezoresistive gauge factor is

$$
G=\frac{\Delta R}{R}\left(\frac{I}{\varepsilon}\right)
$$

where $\varepsilon$ is unitless strain. Table II summarizes the characterized gauge factors for the four electrode configurations. Each mean value in the table was based on three tested cantilevers with the same electrode configuration. The gauge factor of 78.84 to 134.40 obtained in this study is similar to that of piezoresistive cantilevers using doped silicon as piezoresistors with a typical gauge factor lower than 200 [24]-[26]. Compared to the gauge factors of reported CNT-based pressure sensors, 235 for MWNT bundle-based pressure sensors [13] and 210 for single metallic SWNT-based pressure sensors [14], the gauge factor of 78.84 to 134.40 obtained in this study is lower, possibly due to different types of CNTs used for sensor construction.

A gauge factor $\sim 1000$ would permit CNT-based cantilevers to provide more comparable performance as laser detection systems. AFM-based electromechanical characterization results demonstrated that the gauge factor can reach $600-1000$ for 



Fig. 5. Resistance versus strain of CNT-based cantilevers with different electrode configurations.

TABLE II

Statistical VALUES OF GaUge Factors

\begin{tabular}{ccccc}
\hline $\begin{array}{c}\text { Gauge } \\
\text { factor }\end{array}$ & (a) & (b) & (c) & (d) \\
\hline $\begin{array}{c}\text { Mean } \\
\text { Standard } \\
\text { deviation }\end{array}$ & 16.73 & 78.84 & 121.07 & 134.40 \\
\hline
\end{tabular}
(a) Repeated round electrodes
(b) Single round electrodes
(c) Repeated rectangular electrodes
(d) Single rectangular electrodes

individual quasi-metallic SWNTs [12]. However, the difficulty in precisely selecting appropriate CNT types and obtaining uniform electromechanical properties is a hurdle for the development of highly sensitive CNT-based mechanical sensors. Current CNT synthesis techniques are only capable of producing statistical distributions of chiralities, and thus, electrical and electromechanical properties. Continuous progress being made to alleviate these difficulties promises improvements of measurement sensitivity and reproducibility of CNT-based strain sensors such as cantilevers.

The CNT-based cantilevers presented in this paper was based on the batch microfabricated; however, DEP assembly of CNTs was manually conducted in a serial manner. Besides reducing the stiffness of the cantilevers and using individual CNTs as sensing elements, methods such as capacitive coupling [27] can be employed to realize parallel DEP assembly of indi- vidual CNTs toward batch-microfabricated, highly sensitive CNT-based AFM cantilevers.

\section{CONCLUSION}

Utilizing the electromechanical properties of carbon nanotubes, this paper presented microfabricated cantilevers with MWNTs as active strain sensing elements that differ from conventional piezoresistive designs. A batch microfabrication process was developed to construct the cantilevers. MWNTs with an average length of $5 \mu \mathrm{m}$ and an average diameter of $17 \mathrm{~nm}$ were dielectrophoretically assembled between electrodes. The parameter set of $4 \mathrm{MHz}$ and $9 \mathrm{Vp}-\mathrm{p}$ ac signal with a $0.5 \mathrm{~V} \mathrm{dc}$ offset was selected for its highest reproducibility in producing regular MWNT networks with limited number of tubes. The CNT-based cantilevers demonstrated a linear relationship between resistance changes and externally applied strain. The gauge factor ranged from 78.84 to 134.40 for four different electrode configurations. In order to obtain highly sensitive, batch microfabricated CNT-based cantilevers that are directly applicable to AFM strain detection, challenges to tackle include the precise selection of appropriate CNT types (e.g., quasi-metallic SWNTs) and the realization of parallel DEP assembly of individual CNTs.

\section{ACKNOWLEDGMENT}

The authors would like to thank the staff in the microfabrication center at the Emerging Communications Technology Institute of University of Toronto. 


\section{REFERENCES}

[1] J. Hogan, "Molecular microscopy: Focus on the living," Nature, vol. 440, pp. 14-15, Mar. 2006.

[2] J. J. Muys, M. M. Alkaisi, D. O. S. Melville, J. Nagase, P. Sykes, G. M. Parguez, and J. J. Evans, "Cellular transfer and AFM imaging of cancer cells using bioimprint," J. Nanobiotechnol., vol. 4, p. 1, Jan. 2006.

[3] M. F. Yu, O. Lourie, M. J. Dyer, K. Moloni, T. F. Kelly, and R. S. Ruoff, "Strength and breaking mechanism of multiwalled carbon nanotubes under tensile load," Science, vol. 287, pp. 637-640, Jan. 2000.

[4] I. Palaci, S. Fedrigo, H. Brune, C. Klinke, M. Chen, and E. Riedol, "Radial elasticity of multiwalled carbon nanotubes," Phys. Rev. Lett., vol. 94, p. 175 502, May 2005.

[5] J. H. Hafner, C. L. Cheung, and C. M. Lieber, "Growth of nanotubes for probe microscopy tips," Nature, vol. 398, pp. 761-762, Apr. 1999.

[6] C. L. Cheung, J. H. Hafner, T. W. Odom, K. Kim, and C. M. Lieber, "Growth and fabrication with single-walled carbon nanotube probe microscopy tips," Appl. Phys. Lett., vol. 76, no. 21, pp. 3136-3138, May 2000.

[7] H. Dai, J. H. Hafner, A. G. Rinzler, D. T. Colbert, and R. E. Smalley, "Nanotubes as nanoprobes in scanning probe. microscopy," Nature, vol. 384, pp. 147-150, Nov. 1996.

[8] J. Martinez, T. D. Yuzvinsky, A. M. Fennimore, A. Zettl, R. García, and C. Bustamante, "Length control and sharpening of atomic force microscope carbon nanotube tips assisted by an electron beam," Nanotechnology, vol. 16, pp. 2493-2496, 2005.

[9] J. Tang, G. Yang, Q. Zhang, A. Parhat, B. Maynor, J. Liu, L. C. Qin, and $\mathrm{O}$. Zhou, "Rapid and reproducible fabrication of carbon nanotube AFM probes by dielectrophoresis," Nano Lett., vol. 5, no. 1, pp. 11-14, Nov. 2005

[10] T. W. Tombler, C. Zhou, L. Alexseyev, J. Kong, H. Dai, L. Liu, C. S. Jayanthi, M. Tang, and S. Y. Wu, "Reversible electromechanical characteristics of carbon nanotubes under local-manipulation," Nature, vol. 405, pp. 769-772, Jun. 2000.

[11] E. D. Minot, Y. Yaish, V. Sazonova, J. Y. Park, M. Brink, and P. L. McEuen, "Tuning carbon nanotube band gaps with strain," Phys. Rev. Lett., vol. 90, no. 15, p. 156401, Apr. 2003.

[12] J. Cao, Q. Wang, and H. Dai, "Electromechanical properties of metallic, quasi-metallic, and semiconducting carbon nanotubes under stretching," Phys. Rev. Lett., vol. 90, no. 15, p. 157601, Apr. 2003.

[13] C. K. M. Fung, M. Q. H. Zhang, R. H. M. Chan, and W. J. Li, "A PMMA-based micro pressure sensor chip using carbon nanotubes as sensing elements," in Proc. IEEE MEMS 2005, pp. 251-254.

[14] C. Stampfer, T. Helbling, D. Obergfell, B. Schöberle, M. K. Tripp, A. Jungen, S. Roth, V. M. Bright, and C. Hierold, "Fabrication of singlewalled carbon-nanotube-based pressure sensors," Nano Lett., vol. 6, no. 2, pp. 233-237, Jan. 2006.

[15] B. Mahar, C. Laslau, R. Yip, and Y. Sun, "Development of carbon nanotube-based sensors-A review," IEEE Sensors J., vol. 7, no. 2, pp. 266-284, Feb. 2007.

[16] H. T. Soh, C. F. Quate, A. F. Morpurgo, C. M. Marcus, J. Kong, and H. Dai, "Integrated nanotube circuits: Controlled growth and ohmic contacting of single-walled carbon nanotubes," Appl. Phys. Lett., vol. 75, no. 5, pp. 627-629, Aug. 1999.

[17] J. Kong, H. T. Soh, A. M. Cassell, C. F. Quate, and H. Dai, "Synthesis of individual single-walled carbon nanotubes on patterned silicon wafers," Nature, vol. 395, pp. 878-881, Oct. 1998.

[18] A. M. Cassell, N. R. Franklin, T. W. Tombler, E. M. Chan, J. Han, and H. Dai, "Directed growth of free-standing single-walled carbon nanotubes," J. Amer. Chem. Soc., vol. 121, pp. 7975-7976, Aug. 1999.

[19] N. R. Franklin and H. Dai, "An enhanced CVD approach to extensive nanotube networks with directionality," Adv. Mater., vol. 12, no. 12, pp. 890-894, 2000.

[20] T. Fukuda, F. Arai, and L. Dong, "Assembly of nanodevices with carbon nanotubes through nanorobotic manipulations," Proc. IEEE, vol. 91, no. 11, pp. 1803-1818, Nov. 2003.
[21] J. Chung, K. H. Lee, J. Lee, and R. S. Ruoff, "Toward large scale integration of carbon nanotubes," Langmuir, vol. 20, no. 8, pp. 3011-3017, Mar. 2004.

[22] J. Chung and J. Lee, "Nanoscale gap fabrication and integration of carbon nanotubes by micromachining," Sens. Actuators A, Phys., vol. 104, pp. 229-235, 2003.

[23] T. W. Ebbesen, H. J. Lezec, H. Hiura, J. W. Bennett, H. F. Ghaemi, and T. Thio, "Electrical conductivity of individual carbon nanotubes," Nature, vol. 382, pp. 54-56, Jul. 1996.

[24] F. J. Giessibl and B. M. Trafas, "Piezoresistive cantilevers utilized for scanning tunneling and scanning force microscope in ultrahigh vacuum," Rev. Sci. Instrum., vol. 65, no. 6, pp. 1923-1929, 1994.

[25] S. C. Minne, S. R. Manalis, and C. F. Quate, "Parallel atomic force microscopy using cantilevers with integrated piezoresistive sensors and integrated piezoelectric actuators," Appl. Phys. Lett., vol. 67, no. 26, pp. 3918-3920, 1995.

[26] B. W. Chui, T. D. Stowe, Y. S. Ju, K. E. Goodson, T. W. Kenny, H. J. Mamin, B. D. Terris, R. P. Ried, and D. Rugar, "Low-stiffness silicon cantilevers with integrated heaters and piezoresistive sensors for highdensity AFM thermomechanical data storage," J. MEMS, vol. 7, no. 1, pp. 69-78, 1998.

[27] S. Evoy, N. DiLello, V. Deshpande, A. Narayanan, H. Liu, M. Riegelman, B. R. Martin, B. Hailer, J. C. Bradley, W. Weiss, T. S. Mayer, Y. Gogotsi, H. H. Bau, T. E. Mallouk, and S. Raman, "Dielectrophoretic assembly and integration of nanowire devices with functional CMOS operating circuitry," Microelectron. Eng., vol. 75, pp. 31-42, 2004.

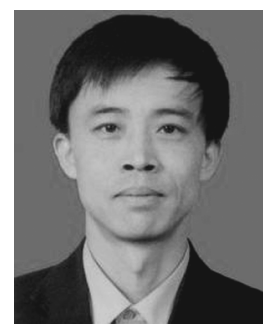

Jianhua Tong (M'07) received the B.S. and Ph.D. degrees from the School of Mechanical Engineering, Dalian University of Technology, China, in 1997 and 2005 , respectively.

$\mathrm{He}$ is currently with the Advanced Micro and Nanosystems Laboratory at the University of Toronto, Toronto, ON, Canada, as a Postdoctoral Research Fellow. His research interests include carbon nanotube-based microsensors, MEMS-based device arrays for studying cellular mechanobiology, and piezoelectric microsensors and microactuators.

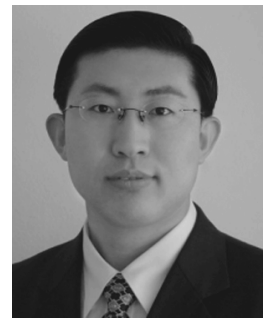

Yu Sun (S'01-M'03-SM'07) received the B.S. degree in electrical engineering from Dalian University of Technology, China, in 1996, the M.S. degree from the Institute of Automation, Chinese Academy of Sciences, in 1999, and the M.S. degree in electrical engineering and the Ph.D. degree in mechanical engineering from the University of Minnesota, Minneapolis, in 2001 and 2003, respectively.

He held a Research Scientist position at the Swiss Federal Institute of Technology (ETH-Zurich). He is currently Assistant Professor of Mechanical and Industrial Engineering Department and is jointly appointed in the Institute of Biomaterials and Biomedical Engineering and Electrical and Computer Engineering Department at the University of Toronto, Toronto, ON, Canada, where he established and directs the Advanced Micro and Nanosystems Laboratory. His research areas are design and fabrication of solid-state MEMS and bioMEMS, microrobotic manipulation of biological cells, cellular biomechanics, nanofabrication, and nanorobotic manipulation of nanomaterials.

Dr. Sun is a recipient of University of Minnesota Dissertation Fellowship and Ontario Early Researcher Award. 